Coherence imaging spectro-polarimetry for magnetic fusion diagnostics

This article has been downloaded from IOPscience. Please scroll down to see the full text article.

2010 J. Phys. B: At. Mol. Opt. Phys. 43144010

(http://iopscience.iop.org/0953-4075/43/14/144010)

View the table of contents for this issue, or go to the journal homepage for more

Download details:

IP Address: 150.203.179.67

The article was downloaded on 21/10/2010 at 04:30

Please note that terms and conditions apply. 


\title{
Coherence imaging spectro-polarimetry for magnetic fusion diagnostics
}

\author{
J Howard \\ Plasma Research Laboratory, Research School of Physics and Engineering, The Australian National \\ University, Canberra, 0200, Australia \\ E-mail: john.howard@anu.edu.au
}

Received 9 February 2010, in final form 9 March 2010

Published 5 July 2010

Online at stacks.iop.org/JPhysB/43/144010

\begin{abstract}
This paper presents an overview of developments in imaging spectro-polarimetry for magnetic fusion diagnostics. Using various multiplexing strategies, it is possible to construct optical polarization interferometers that deliver images of underlying physical parameters such as flow speed, temperature (Doppler effect) or magnetic pitch angle (motional Stark and Zeeman effects). This paper also describes and presents first results for a new spatial heterodyne interferometric system used for both Doppler and polarization spectroscopy.
\end{abstract}

(Some figures in this article are in colour only in the electronic version)

\section{Introduction}

Static and electro-optically modulated polarization interferometers for wide-field time-resolved 'coherence-imaging' can obtain two-dimensional projections (images) of key spectral quantities in Doppler spectroscopy (including chargeexchange recombination spectroscopy - CXRS) and polarization spectroscopy [1-4]. For a sufficiently simple spectral scene, the physical parameters determining the emission spectral properties can often be recovered from measurements of the complex coherence (phase and amplitude of the interferogram) at a small number of appropriately chosen fixed optical path length delays. For example, for Doppler spectroscopy, measurement of the optical coherence can be related to projections of parameters such as brightness-weighted temperature and flow speed [3]. This paper reviews the various schemes and multiplex strategies that have been adopted to enable timeresolved imaging of important physical parameters conveyed by the spectral properties of optical emission from plasmas. A new spatial-heterodyne multiplex system is also introduced here, and first results for Doppler imaging are presented.

In its simplest form, a polarization interferometer is constructed of a suitable birefringent delay plate with its fast axis oriented at $45^{\circ}$ to, and sandwiched between, crossed or parallel polarizers. An optical interference pre-filter is used to transmit only the spectral region of interest. Polarization interferometers naturally facilitate various multiplex strategies and, because there are no slits, allow for two-dimensional imaging of key spectral features using robust components with high optical throughput.

One approach to extract multi-channel optical coherence information is to use temporal or temporal-frequency domain multiplex techniques. In the latter case an electro-optically path-modulated polarization interferometer allows the targeted coherence information to be encoded over a spread of harmonics of the modulation frequency [5]. These systems can be very fast ( $>\mathrm{MHz}$ ), with temporal resolution usually being limited only by the available light flux. Temporal multiplex methods can also be adapted to $2 \mathrm{D}$ imaging using CCD cameras, with the interferometer path difference electrooptically stepped synchronously with the camera frame rate [6]. In this case, extraction of useful information requires typically three successive frames obtained at different path lengths in order to recover fringe brightness, contrast and phase. Fast CMOS cameras combined with ferro-electric switches or electro-optic crystals allow very high frame rates up to $10 \mathrm{kHz}$ or more to be achieved.

A completely static approach that utilizes a pair of Wollaston prisms to produce independent images of the optical coherence in four quadrants of the CCD array has been reported in [7]. Because the quadrature coherence images are sampled simultaneously, the static arrangement allows the capture and imaging analysis of fast transient plasma events. Combined with field-of-view widening techniques, polarization manipulation and suitable imaging optics, it is possible to perform fast, high-throughput, time-resolved 2D imaging of plasma parameters that affect the emitted spectrum. 
A new static spatial heterodyne approach which, depending on the application, offers a number of advantages over previous implementations is introduced here. A birefringent Savart plate is used to produce an angle-dependent interferometric phase shear to generate approximately parallel spatial interference fringes in the image plane. The fringe phase and amplitude can be demodulated and compared with the properties of fringes produced by a reference monochromatic light source. This system has been optimized for imaging Doppler spectroscopy of flows in low-field discharges in the $\mathrm{H}-1$ heliac, a medium-sized 3-period helical axis stellarator at the Australian National University $[8,9]$. The instrument shares some common features with spatial heterodyne spectro-polarimetry for motional Stark Effect (MSE) imaging — a new polarimetric imaging modality recently tested in experiments on the TEXTOR tokamak $[4,10]$.

The paper is organized as follows. Section 2 establishes the link between the properties of the interferogram and the Doppler shift and broadening of an isolated spectral line or multiplet. In the important case of an inhomogeneous radiating medium (e.g. in the divertor region of a tokamak) we establish invertible relations between the interferometric measurements and spatially varying distributions of brightness, temperature and flow speed. The key features of imaging polarization interferometers for Doppler spectroscopy are presented in section 3. For completeness, this section also briefly reviews the operating principles for previously reported time-domain-multiplex and quadrature-multiplex coherence imaging systems. Section 4 introduces the spatial heterodyne coherence imaging system as applied to Doppler spectroscopic imaging of flows in the $\mathrm{H}-1$ heliac. Finally, section 5 summarizes the extension of spatial heterodyne techniques to polarimetric motional Stark effect imaging and shows results confirming the expected Doppler phase shift when observing the $50 \mathrm{keV}$ injected neutral beam on the TEXTOR tokamak.

\section{Doppler spectroscopy and interferometry}

In this section we obtain some basic results linking the observed optical coherence to the properties of the velocity distribution function of the radiating species. For simplicity, we consider first a homogeneous medium so that line-of-sight integration effects can be ignored.

The Fourier transform spectrometer (interferometer) splits an incident scalar wave component $u(t)$, relatively delaying the nominally equal amplitude components by time $\tau$ before they are recombined at a square-law detector. The intensity of the transmitted or reflected light at the final beam combiner is proportional to [11]

$$
S_{ \pm}(\phi)=\frac{I_{0}}{2}[1 \pm \Re[\Gamma(\tau)]]
$$

where $I_{0}=\left\langle u u^{*}\right\rangle$ is the spectrally integrated irradiance, or brightness, and $\Gamma$ is the complex coherence given by $\Gamma=\left\langle u(t) u^{*}(t+\tau)\right\rangle / I_{0}$ where angle brackets denote a time average. The complex temporal coherence is related to the spectral distribution of the irradiance $I(v)$ through the WienerKhinchine theorem

$$
\Gamma(\tau)=\frac{1}{I_{0}} \int_{-\infty}^{\infty} I(\nu) \exp (\mathrm{i} 2 \pi \nu \tau) \mathrm{d} \nu .
$$

For quasi-monochromatic radiation, the field $u$ can be expressed in terms of the analytic signal

$$
u(t)=A(t) \exp \left(-\mathrm{i} 2 \pi v_{0} t\right)
$$

where the complex amplitude $A$ changes slowly compared with the exponential and $v_{0}$ is the light centre frequency [12]. In terms of the phase delay $\phi_{0}=2 \pi v_{0} \tau$, the intensity of the transmitted or reflected light at the final beam combiner becomes

$$
S_{ \pm}\left(\phi_{0}\right)=\frac{I_{0}}{2}\left[1 \pm \Re\left[\gamma\left(\hat{\phi}_{0}\right) \exp \left(\mathrm{i} \phi_{0}\right)\right]\right]
$$

where $\gamma\left(\hat{\phi}_{0}\right)$ is the temporal coherence of the spectral line. Note that $\gamma$ is a function of the group phase delay coordinate $\hat{\phi}_{0}=\kappa \phi_{0}$, where

$$
\kappa=1+\left.\frac{\nu_{0}}{\tau_{0}} \frac{\partial \tau}{\partial v}\right|_{\nu_{0}}
$$

accounts for any optical frequency dispersion of the time delay.

To illustrate the properties of the interferogram, we introduce the spectral line shape $g$ characterized by the normalized spectral line shift $\xi_{D}=v_{D} / \nu_{0}$ and width $\sigma=$ $\Delta v / v_{0}$

$$
I(\xi)=I_{0} g\left(\frac{\xi-\xi_{D}}{\sigma}\right)
$$

with a dimensionless frequency coordinate $\xi=\left(v-v_{0}\right) / v_{0}$. Using equations (2) and (4), the interferogram at fixed optical delay $\phi_{0}$ is calculated as

$$
S_{ \pm}=\frac{I_{0}}{2}\left[1+\zeta \cos \left(\phi_{0}+\phi_{D}\right)\right]
$$

where $I_{0}$ is the emission intensity and for a spectral profile having even symmetry, the fringe visibility, or contrast $\zeta \equiv|\gamma|$, and the phase shift $\phi_{D}$ are given by

$$
\begin{gathered}
\zeta=G\left(\sigma \hat{\phi}_{0}\right) \\
\phi_{D}=\hat{\phi}_{0} \xi_{D}
\end{gathered}
$$

where $G(\phi)$ is the Fourier transform of the line shape $g(\xi)$.

Observe that wavelength shifts $\xi_{D}$ scale the interferogram phase but leave the fringe visibility $\zeta$ essentially unchanged. On the other hand, when the spectral width $\sigma$ changes, only the fringe visibility is affected, the underlying phase remaining undisturbed. Measurements of the interferogram at one or more fixed delay offsets $\phi_{0}$ are sufficient to characterize a spectrum that can be described in terms of a small number of parameters. 


\subsection{Drifting local thermal equilibrium}

With the simplifying assumption that the plasma is in drifting local thermal equilibrium with local drift velocity $\boldsymbol{v}_{D}(\boldsymbol{r})$, the spectral line shape in some direction $\hat{l}$ is given by

$$
g(\boldsymbol{r}, \xi)=\left(\pi \xi_{\mathrm{th}}^{2}\right)^{-1 / 2} \exp \left[-\left(\xi-\xi_{D}\right)^{2} / \xi_{\mathrm{th}}^{2}\right]
$$

where $\boldsymbol{r}$ is a position in the plasma, $\xi_{D}=\boldsymbol{v}_{D} \cdot \hat{\boldsymbol{l}} / c$ and $\xi_{\text {th }} \equiv v_{\text {th }}(\boldsymbol{r}) / c$ is the local Doppler thermal width. Since the exponent involves $\boldsymbol{r}$-dependent unknowns $v_{D}(\boldsymbol{r})$ and $\xi_{\text {th }}$, and because the optical-frequency-domain measurement also convolves $g(\boldsymbol{r}, \xi)$ with the instrument spectral response, the tomography of the spectral irradiance presents a difficult inverse problem.

The Fourier transform of the line shape in the locally drifting frame takes the form

$$
G(\phi)=\exp \left(-\hat{\phi}^{2} \xi_{\mathrm{th}}^{2} / 4\right) .
$$

For thermally broadened spectral line emission from a homogeneous plasma, the Doppler phase shift $\phi_{D}$ is proportional to the component $v_{D}$ of the species flow speed in the direction of the line-of-sight (equation (9)) while the fringe contrast $\zeta \equiv \zeta_{D}$ at a fixed delay offset $\phi_{0}$ depends inversely on the optical line width

$$
\zeta_{D}=\exp \left(-T_{S} / T_{C}\right)
$$

where $T_{S}$ is the species temperature and $T_{C}$ is the instrument 'characteristic temperature':

$$
k T_{C}=2 m_{S} c^{2} / \hat{\phi}_{0}^{2} .
$$

For optimum sensitivity to temperature changes, the characteristic temperature (wave-plate thickness) is chosen to be close to the expected source temperature (requiring that the group delay satisfy $\hat{\phi}_{0} v_{\mathrm{th}}=2$ ).

\subsection{Inhomogeneous plasmas}

As shown in figure 1, the spectral line shape is the sum of intensity weighted Doppler-shift contributions from all plasma positions along the line-of-sight. While the general case of inhomogeneous media is discussed elsewhere [13], we reproduce here key results in the case of an inhomogeneous plasma in drifting local thermal equilibrium.

Provided that the Doppler phase shift $\hat{\phi}_{0} \xi_{D}$ is small, the modulus of the complex coherence (the fringe visibility, or contrast) which characterizes the inhomogeneous distribution is insensitive in first order to the drift:

$$
\zeta_{D} \equiv|\gamma(\phi ; \hat{\boldsymbol{l}})|=\frac{1}{\mu_{0}} \int_{L} I_{0}(\boldsymbol{r}) \exp \left[-T_{S}(\boldsymbol{r}) / T_{C}\right] \mathrm{d} l
$$

where

$$
\mu_{0}=\int_{L} I_{0}(\boldsymbol{r}) \mathrm{d} l
$$

is the line-integrated brightness (for simplicity, we ignore issues related to collection solid angle). When the species temperature changes, only the fringe visibility is affected, the underlying phase remaining undisturbed. The fringe visibility can be inverted by standard tomographic techniques to give a scalar function of the local species temperature.

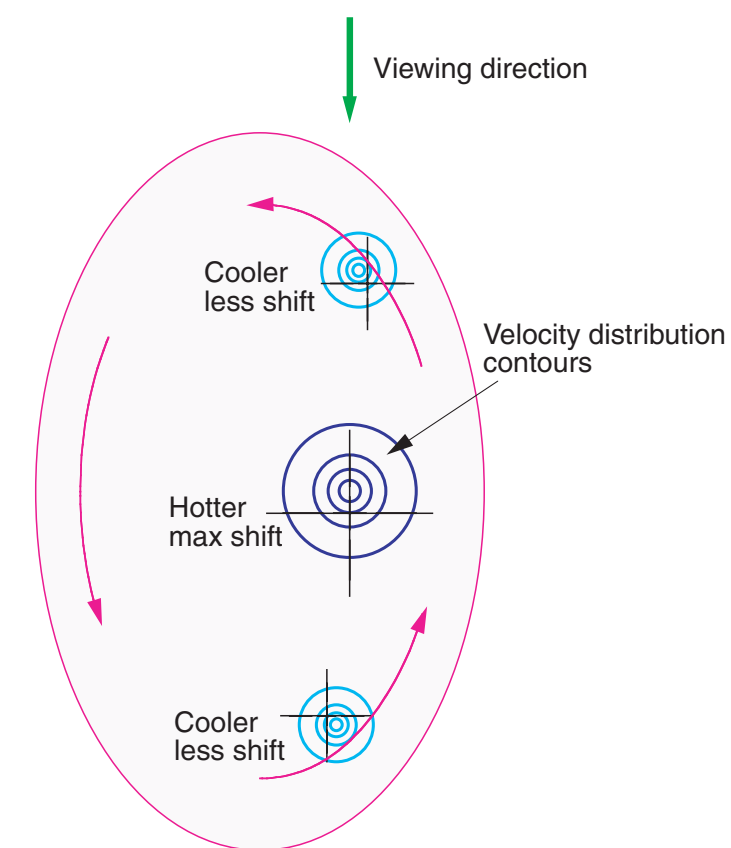

Non-Gaussian, shifted asymmetric lineshape

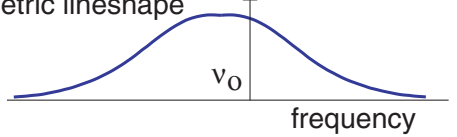

Figure 1. The spectral line shape is the sum of intensity weighted Doppler-shifted contributions from all plasma positions along the line-of-sight.

Again, provided that $\hat{\phi}_{0} \xi_{D}$ is small, a quantity related to the first spectral moment can be obtained from the phase of the complex coherence

$$
\frac{\phi_{D}}{\hat{\phi}_{0}}=\frac{1}{\mu_{0} c|\gamma|} \int_{L} I_{0}(\boldsymbol{r}) \exp \left[-T_{S}(\boldsymbol{r}) / T_{C}\right] \boldsymbol{v}_{D} \cdot \mathrm{d} \boldsymbol{l} .
$$

The instrumental phase offset $\phi_{0}$ is chosen to magnify the usually small Doppler shift component but not so large as to invalidate our first-order approximation. In response to small variations in the local drift speed $\boldsymbol{v}_{D}$, the interferogram fringe pattern expands or contracts as the phase changes, leaving the fringe visibility unchanged. This is a consequence of the Fourier shift theorem. The decoupling of these influences on the phase and envelope of the interferogram is one of the more important advantages offered by time-domain spectroscopic methods applied to Doppler tomography. Finally, it is apparent that the interferometric phase gives longitudinal line integral measurements of the vector field $I_{0} G_{0} \boldsymbol{v}_{D}$ and so is amenable to tomographic inversion for the $z$ component of the flow vector potential [14].

\section{Polarization interferometers for Doppler spectroscopy}

The simplest polarization interferometer (see figure 2) is constructed from a single fixed-delay wave plate sandwiched between parallel or crossed polarizers and with its fast axis at $45^{\circ}$ to the orientation of the polarizers. The plate mutually 


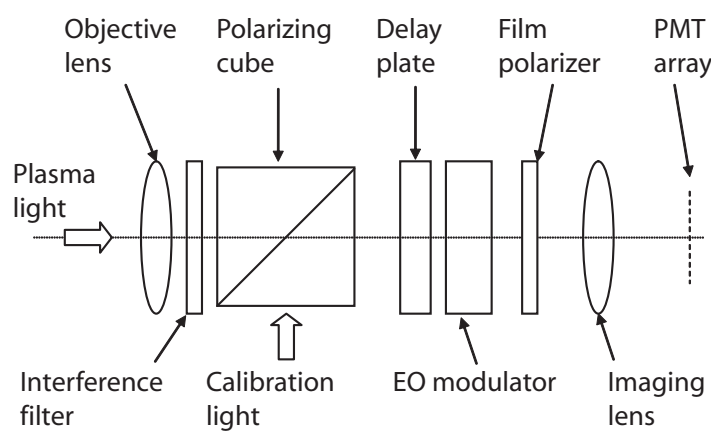

Figure 2. Optical layout for a modulated fixed-delay imaging polarization interferometer.

delays polarization components parallel and perpendicular to the fast axis by $\tau=L B(v) / c$ where $L$ is the thickness of the plate, $B(v)=n_{E}-n_{O}$ is the plate birefringence and $n_{E}$ and $n_{O}$ are the extraordinary and ordinary wave refractive indices respectively. The associated phase delay is $\phi_{0}=2 \pi v_{0} \tau$, while the group delay $\hat{\phi}_{0}$ can be estimated using the Sellmeier equations appropriate for the birefringent delay plate (see equation (5)). For example, for a lithium niobate delay plate at $468.6 \mathrm{~nm}$ the group delay coefficient is $\kappa \simeq 1.6$. It is clear that polarization interferometers are not subject to the mechanical and acoustic noise problems normally associated with optical Fourier transform spectrometers.

In Doppler spectroscopy applications, the ability of the interferometer to capture changes in fringe contrast depends on the optical phase delay introduced by the birefringent plate. For example, a spectral line of width $0.1 \mathrm{~nm}$ and centred at $500 \mathrm{~nm}$ requires a plate producing a delay of roughly $\hat{\phi}_{0} / 2 \pi \sim 5000$ waves in order to be sensitive to changes in the optical coherence length (see equation (8)). For optimum sensitivity to temperature changes, one chooses the characteristic temperature $T_{C}$ to be comparable with the expected species temperature $T_{S}$. Though narrow line widths require a large optical delay, this can be achieved with little cost to light throughput. The instrument dynamic range for both temperature and flow speed variations depends on the light flux, but in practice typically spans an order of magnitude [2]. Given that the resolution of a grating spectrometer is inversely proportional to the entrance and exit slit widths, the light throughput advantage for interferometers is most evident in high-resolution applications.

The interferometer instrument response can be measured by illuminating the field of view with a suitably diffused monochromatic light source (laser beam or spectral lamp) having wavelength at or near the wavelength of interest. Issues related to characterization of the instrument function and performance are presented in detail elsewhere [2]. Degradation of instrument contrast with increasing collection solid angle through the delay plates can be remedied by interposing a half wave plate between two identical crossed delay plates [15]. The resulting field-of-view is expanded by a factor $[2 \sqrt{2} n / B]^{1 / 2}$ to typically tens of degrees or more.

It is necessary to scan or modulate the interferometric path difference in order to measure the fringe contrast and phase at the chosen phase delay offset $\phi_{0}$. Polarization interferometers naturally present a number of path length modulation strategies. For example, if the wave plate is also electro-optic (e.g. $\mathrm{LiNbO}_{3}$ ), the application of a high voltage along an appropriate crystal axis of the wave plate will allow the optical path delay to be varied in order to measure the local fringe properties. In the following sections we describe a number of instruments that implement different path-length modulation/multiplex strategies, the relative merits depending on the experimental context.

\subsection{Temporal frequency domain multiplex interferometers}

We have constructed a number of 1D and 2D time domain multiplex imaging systems for plasma cross-sectional imaging and tomography. The interferometers are coupled to both linear and 2D multi-anode phototube arrays and fast framing CCD cameras. The cameras use standard F-mount lenses and $50 \mathrm{~mm}$ optics with typically $\sim 40 \mathrm{~mm}$ clear aperture to match that of the birefringent plates. The systems are modular in construction, using interchangeable flanged components that can be bolted to an optical rail. Such coherence imaging systems have been installed and operated on the $\mathrm{H}-1$ heliac $[16,17]$, at the RFX reversed field pinch in Padova, Italy and at the WEGA stellarator in the Max Planck Institute for Plasma Physics in Greifswald, Germany [6].

A simple way to measure the coherence at delay $\phi_{0}$ is to sinusoidally modulate the optical path delay by $\phi_{1} \gtrsim \pi / 2$ so that the total time-varying interferometric phase is given by

$$
\tilde{\phi}_{0}=\phi_{0}+\phi_{1} \sin \Omega t \text {. }
$$

This approach encodes the coherence information over a spread of harmonics of the modulation frequency with measurement bandwidth equal to half the modulation frequency [5]. The delay modulation $\tilde{\phi}_{1}=\phi_{1} \sin \Omega t$ can be conveniently obtained by applying an oscillating electric field along the electro-optic crystal $Z$-axis transverse to the direction of the light propagation. Though both the modulation and delay offset can often be obtained using the same optical component [16], in practice it is generally more convenient to separate these functions. When using phototube detectors, light signals are generally low-pass filtered and amplified prior to synchronous data acquisition using $4 \mathrm{f}$, $8 \mathrm{f}$ or $16 \mathrm{f}$ synchronous external clocks, depending on the spread of harmonic carriers.

This system has been used to study the evolution of the ion distribution function and dynamical radial force balance in rf-sustained (7 MHz, $80 \mathrm{~kW}$ ) discharges in argon at low magnetic field strengths $(0.1 \mathrm{~T})$ in the $\mathrm{H}-1$ heliac. Low field results are based on 1D measurements in a poloidal crosssection of Doppler broadening of the Ar II transition at $488 \mathrm{~nm}$. Observed ion temperatures are in the range $5-20 \mathrm{eV}$, increasing with magnetic field strength and decreasing with neutral fill pressure, with radial profiles that are found to be flat or slightly hollow. At small radii, the plasma poloidal flow speed is consistent with rigid rotation (proportional to radius, and reversing about the plasma axis). The flow reaches a typical maximum speed of around $1000 \mathrm{~m} \mathrm{~s}^{-1}$ at the plasma mid radius before dropping to low values at the plasma boundary. Typical profiles (obtained using other techniques) are shown in figure 6. 


\subsection{Temporal multiplex $2 D$ imaging systems}

The temporal-multiplex 2D coherence imaging system couples a step-modulated fixed delay Fourier transform spectrometer to a fast framing CCD camera $[3,6]$. Crossed Y-cut electrooptic, $10 \mathrm{~mm}$ thick, lithium tantalate plates have been used to produce the optical path-length modulation for this system. More recently, however, we have found it convenient to replace the high voltage modulator and associated hardware with dual fast switching ferroelectric liquid crystal wave plates. These half and quarter wave devices are aligned such that their optical axes in the unswitched state are parallel to those of the primary wave plate. In this case the plates contribute an additional three quarters of a wave delay to the path delay introduced by the primary wave plate. With the application of a low bias voltage of order $10 \mathrm{~V}$, the FLC axes rotate by $45^{\circ}$ so as to be parallel to the polarizers and their associated delay no longer contributes to the interferometer. The FLC cells, which can switch at speeds up to $20 \mathrm{kHz}$, are synchronized with the camera, and temporally interleaved, to produce co-, quadrature and antiphase images of the interferogram. Though it is assumed that the plasma is quasi-stationary over the time required to acquire the images, an iterative processing algorithm can accommodate slow changes.

Doppler studies of the $468.6 \mathrm{~nm}$ He II emission on the WEGA stellarator have been reported by Chung [6]. In these low-temperature experiments, the He II $n-n^{\prime}=4-3$ transition at $468.6 \mathrm{~nm}$ exhibits a complex fine structure. To properly interpret the contrast changes due to Doppler broadening, it is necessary to properly model the interferogram produced by the superposition of Zeeman-split and Dopplerbroadened multiplet components. The calculated multiplet structure for the Doppler- and Zeeman-broadened He II $468 \mathrm{~nm}$ emission line and measurement results for a variety of conditions are reported in [6].

\subsection{Quadrature imaging systems}

A polarization interferometer that multiplexes independent interferometric images to four quadrants of a CCD array has been devised to enable single snapshot 2D spectral imaging [7]. Angular multiplex techniques based on Wollaston prisms and image plane masks are used to produce four images with distinct optical path delays. To achieve this, the first interferometer polarizer is replaced by a Wollaston prism that produces angularly displaced, orthogonally polarized beams that traverse separate paths through effectively independent interferometers. A lens following this first Wollaston produces dual images, say $I_{\text {top }}$ and $I_{\text {bottom, at the first, intermediate }}$ imaging plane (see figure 3). These overlapping images can be isolated using a split-field-polarizer mask, constructed of adjoining, orthogonally oriented polarizers. The relative optical delay of the dual images can also be manipulated at the mask. For example, to produce quadrature images, a low-order quarter wave plate can be inserted into one of the independent polarized images.

Following the mask, rays from the separate, orthogonally polarized images are collimated by a second lens before traversing the remaining interferometer optics. The two
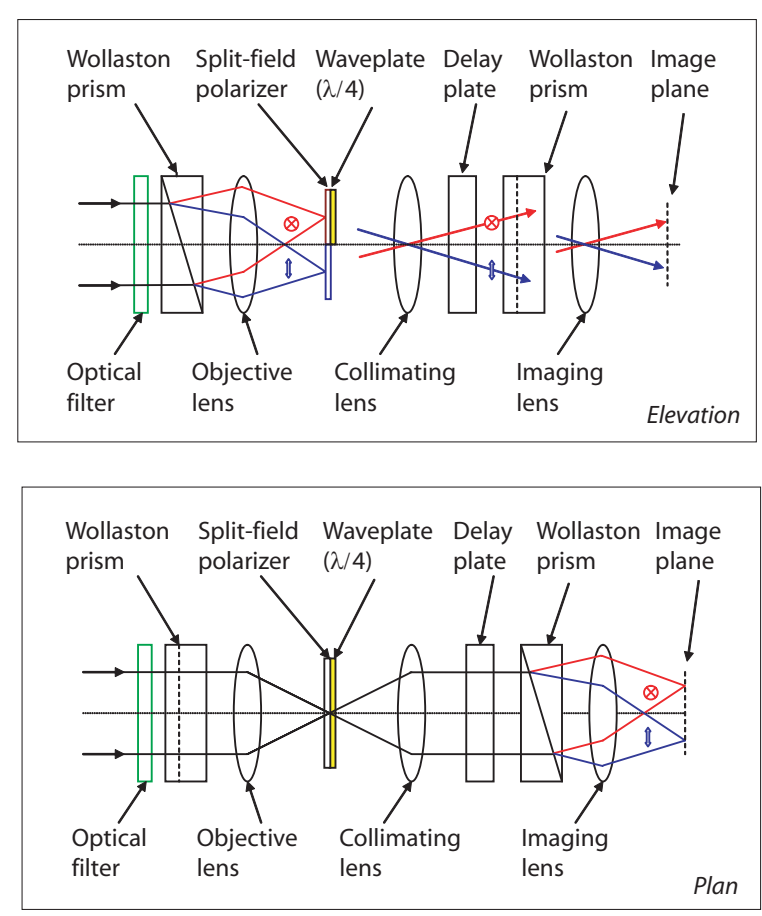

Figure 3. Plan and elevation views of the optical layout for producing four-quadrant coherence images of a quasi-monochromatic source. See also [7].

images act as independent light sources with the angularly separated beams traversing a common birefringent delay plate before encountering a second and final polarizing Wollaston prism oriented to split the beams in a horizontal direction (orthogonal to the first prism). In conjunction with an imaging lens, four separate and independent images are formed at the four corners of the CCD array. For each of $I_{\text {top }}$ and $I_{\text {bottom, }}$, the left and right interferometric images, $S_{+}$and $S_{-}$, can be summed to give the total brightness image $S_{+}+S_{-}$. The difference image normalized to the sum $\left(S_{+}-S_{-}\right) /\left(S_{+}+S_{-}\right)$ gives the co-phase component of the complex coherence for each of $I_{\text {top }}$ and $I_{\text {bottom }}$.

If a quarter wave delay is introduced into one of the images $I_{\text {top }}$ or $I_{\text {bottom }}$ at the intermediate image plane, the resulting four images of the optical coherence will reside in quadrature about a common fixed optical delay offset. The four-quadrant image thereby gives a snapshot of the local interferogram phase and contrast without recourse to temporal multiplexing. This arrangement facilitates very fast or synchronous imaging of simple spectral scenes using, for example, a gated intensified camera.

Measurements of low-field argon plasma discharges, similar to those reported above for the electro-optically modulated camera, have confirmed the 2D imaging performance of the static quadrature-multiplex system [7].

\section{Spatial heterodyne coherence imaging systems}

While the quadrature imaging system can capture the coherence information in a single snapshot, it is a relatively intricate optical arrangement that requires careful system 


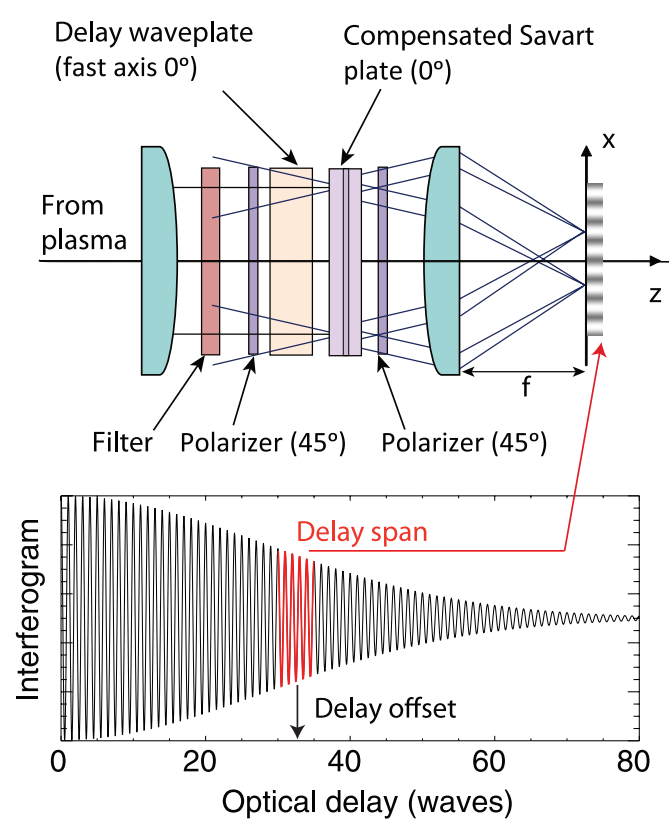

Figure 4. Top: optical arrangement for producing spatial heterodyne images of a Doppler-broadened spectral line. The delay plate at $0^{\circ}$ resolves the polarized input into mutually delayed vertically and horizontally polarized components. These are spatially separated vertically using a zero-nett-delay compensated Savart plate. When imaged through an analyser at $45^{\circ}$, the vertical separation gives rise to an angular shear in the mutual phase delay to produce horizontally aligned interference fringes. Bottom: the initial delay offset is produced by the delay plate while the bolded part of the interferogram corresponds to the spatial heterodyne fringes formed in the focal plane of the final lens.

calibration and image registration procedures. In this section we describe a simpler shearing polarization interferometer combined with an optical telescope that encodes the coherence information upon a one-dimensional image plane interference fringe pattern which is formed by the plasma light. A birefringent Savart plate is used to produce the angledependent interferometric phase shear necessary to generate approximately parallel spatial interference fringes in the lens focal plane. One can consider the interference fringes as being produced by a sinusoidal spectral filter that shifts with image angle across the multiplet spectrum to give rise to regions of alternating high and low relative brightness. The phase and amplitude of the fringe pattern can be demodulated and compared with the properties of fringes produced under illumination by a reference monochromatic light source.

To describe the optical system in more detail, light is assumed to be propagating in the $z$-direction, parallel to the axis of the optical system with the observed multiplet isolated from other spectral features using a suitably chosen interference filter. The first element in the optical chain (see figure 4 ) is a polarizer oriented at $45^{\circ}$ followed by a fixed delay, preferably a field-widened delay plate that sets the bias optical delay offset. The wave plate has its fast axis oriented at $0^{\circ}$ (horizontally or vertically) and the delay (in waves) is chosen to be of the order of the inverse of the normalized spectral width.

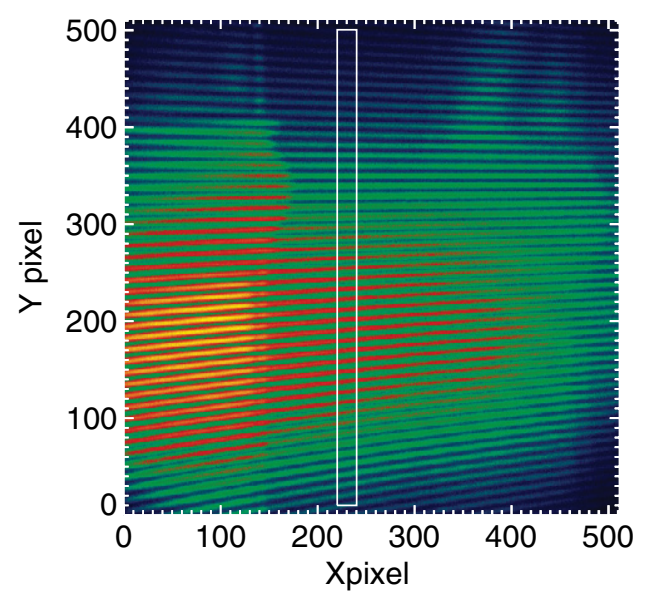

Figure 5. Typical snapshot Doppler image of argon ion radiation at $488 \mathrm{~nm}$ in the H-1 heliac. Toroidal field coils are visible in the left and right sides of the image. The fringes are demodulated and compared with reference fringes obtained using a calibration light source at or near the plasma emission wavelength. Profiles extracted from the rectangular region of interest are presented in figure 6 .

The delay plate is followed by a field-widened Savart prism (see the appendix) that spatially separates the horizontal and vertical polarization components in the $x$-direction. A final analyser oriented at $45^{\circ}$ and a lens cause the polarized and mutually delayed wave fields to interfere to produce a horizontal fringe pattern in the focal plane at the detector array. The path difference at a vertical position $x$ from the origin in the focal plane depends on the Savart plate thickness (beam separation $d$ ) and the lens focal length according to (see appendix equation (A.5)) $\Delta=x d / f$. As a result, the interferometric phase consists of contributions from both wave plate and Savart shearing plates $\phi=\phi_{0}+\phi_{S}$ with $\phi_{S}=2 \pi x d /\left(f \lambda_{0}\right)$. The overall group delay becomes $\hat{\phi}_{0}+\hat{\phi}_{S}$ where $\hat{\phi}_{S}=\kappa_{S} \phi_{S}$ and the optical frequency dispersion of the Savart delay is given by equation (A.6). In practice, for zeronett-delay compensated Savart prisms, $\left|\hat{\phi}_{S}\right| \ll\left|\hat{\phi}_{0}\right|$ and the Savart delay can be neglected.

Figure 5 shows a typical snapshot spatial heterodyne Doppler image of argon ion radiation at $488 \mathrm{~nm}$ produced in low-field $(0.1 \mathrm{~T})$ radio-frequency-heated $(7 \mathrm{MHz}, 60 \mathrm{~kW})$ argon plasmas in the $\mathrm{H}-1$ heliac. The structures to the left and right of the image are reflections from the inside surfaces of the toroidal field coils. The faint circular fringe pattern is produced by the narrowband $488 \mathrm{~nm}$ interference filter. The primary horizontal fringe pattern is locally demodulated for plasma Doppler parameters. The divergence of the fringe pattern is due to the natural hyperbolic phase pattern produced by the delay plate which was not compensated (no field widening) for these measurements.

Profiles of inferred flow speed and fringe contrast averaged across the region of interest depicted in figure 5 are shown in figure 6 . The peak fringe contrast of $\sim 0.5$ corresponds to an argon ion temperature of $5 \mathrm{eV}$, with the apparent temperature increasing slightly towards the edge of the plasma. Together with the sheared flow speed profile, these 


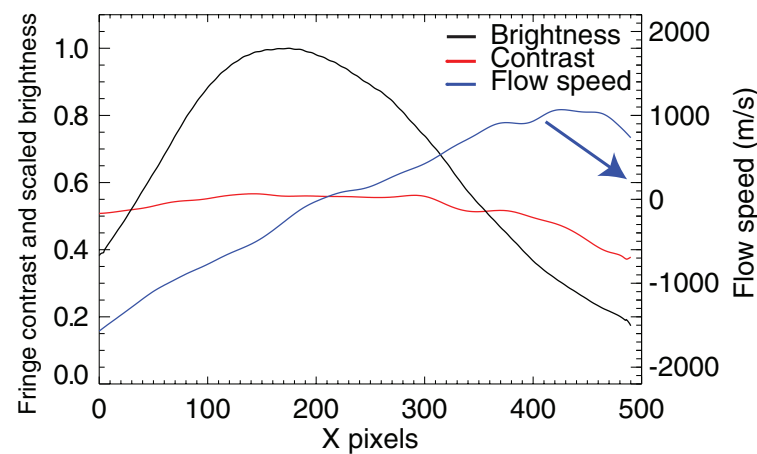

Figure 6. Profiles of normalized brightness, fringe contrast and inferred flow speed averaged across the rectangular region of interest shown in figure 5 .

observations are consistent with those reported in previous works (e.g. [18]).

\section{Spatial heterodyne motional Stark effect polarimetry}

Motional Stark effect polarimetry is a routine diagnostic for high power fusion devices that employ diagnostic or heating neutral beams. Measurement of the polarization state of the Stark split multiplet is used to infer the internal toroidal current density profile [19, 20]. Until now, because of various technical limitations, especially in low-field compact systems where the Stark multiplet is difficult to resolve or is contaminated by other spectral features, MSE systems have been limited to 10 or 20 discrete channels viewing positions across the injected beam.

The MSE technique relies on the splitting of the Dopplershifted neutral beam Balmer $\alpha$ light into orthogonally polarized $\sigma$ and $\pi$ components as a result of the motioninduced electric field $\boldsymbol{E}=\boldsymbol{v} \times \boldsymbol{B}$ experienced in the rest frame of the neutral atoms. Observation at an angle to the energetic beam, conveniently Doppler shifts the multiplet away from the background $\mathrm{H}_{\alpha}$ radiation. When viewed in a direction perpendicular to $\boldsymbol{E}$ the Stark split $\sigma$ and $\pi$ components are polarized respectively perpendicular and parallel to the direction of $\boldsymbol{E}$. When viewed along $\boldsymbol{E}$ the $\sigma$ components are unpolarized and the $\pi$ components have no brightness. The Stark separation of adjacent Balmer alpha spectral components varies as $\Delta \lambda_{S}=2.7574 \times 10^{-8} E \mathrm{~nm}$ where $E=|\boldsymbol{v} \times \boldsymbol{B}|$ is the induced electric field [21]. Integrated over wavelength, the Stark multiplet is nett unpolarized and no orientational information can be obtained.

The magnetic field pitch angle is usually estimated by isolating and measuring the polarization direction of the central cluster of $\sigma$ lines. This requires a tunable narrow-band filter to spectrally resolve the multiplet in order to obtain a nett polarization that is analysed by a suitable polarimeter. Every spatial channel thus requires a dedicated filter whose passband must be optimized by tilt or thermal tuning. Recent developments pioneered at the ANU have allowed, for the first time, 2D imaging of the internal magnetic field of tokamak fusion devices [4]. This is achieved using a spatial heterodyne

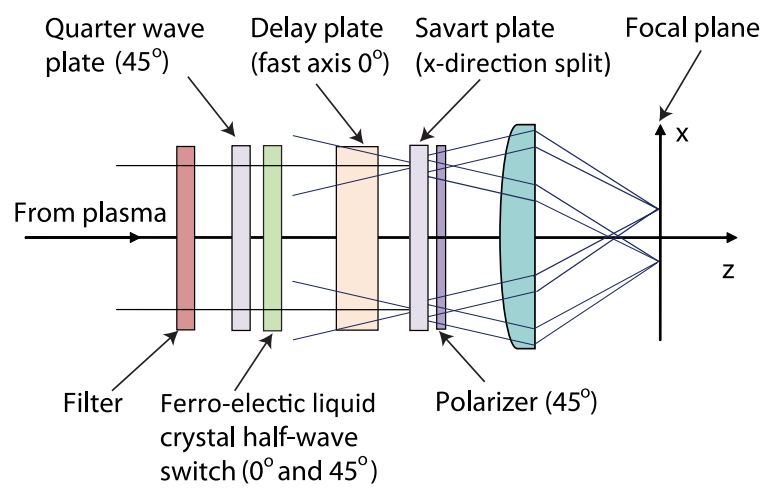

Figure 7. Layout of the switching spectro-polarimeter. The quarter wave plate and FLC switch constitute the polarimeter. The primary delay plate and polarizer provide the spectral discrimination necessary to ensure a non-zero nett polarization. The Savart plate imprints a sinusoidal spatial carrier wave in the $x$-direction.

polarization interferometer to imprint the optical coherence (spectral) and polarimetric information on orthogonal carrier fringe patterns.

In the slightly simpler scheme shown in figure 7 , a 1D fringe pattern imprinted on the neutral beam image provides spectral discrimination in the same fashion as for the spatial heterodyne interferometer described in section 4 . The contrast and phase of the fringes depend on the spectral separation of the Stark components and their mean wavelength respectively. A front end polarimeter which employs a switching liquid crystal wave plate introduces an additional phase offset in proportion to the local polarization orientation of the entire Stark multiplet. The phase difference between successive independent images gives an image of the local magnetic field pitch angle while the mean phase carries the beam Doppler offset. A full description of this hybrid spatio-temporal multiplex imaging system along with initial polarimetric results obtained on the TEXTOR tokamak are reported elsewhere [10].

Figure 8(a) shows the interior of the TEXTOR vacuum vessel as viewed by the spectro-polarimeter while figure $8(\mathrm{~b})$ shows a representative image of neutral beam as transmitted through the $662 \mathrm{~nm}$ interference filter. The spatial fringes, which appear only in the presence of polarized emission, have been oriented parallel to the horizontal midplane in order to maximize the radial spatial resolution. The fringe properties are recovered by demodulating the images column-by-column and comparing with a reference interferogram produced by a hydrogen Balmer-alpha lamp.

Because of the spatial fringe encoding and the ability to accept a relatively wide spectral window, it becomes feasible to undertake two-dimensional polarimetric imaging. The interpretation of the 2D polarimetric MSE images has been investigated in detail elsewhere [22]. For off-axis imaging, it is clearly important to know the spatial distribution of beam velocities which generate the induced electric field. This can be self-consistently captured by the Doppler-shifted phase of the spatial heterodyne carrier fringes. This phase shift is obtained from equation (9) as $\phi_{D}=\hat{\phi}_{0}\left(v_{B} / c\right) \hat{\boldsymbol{v}}_{B} . \hat{\boldsymbol{l}}$ where 


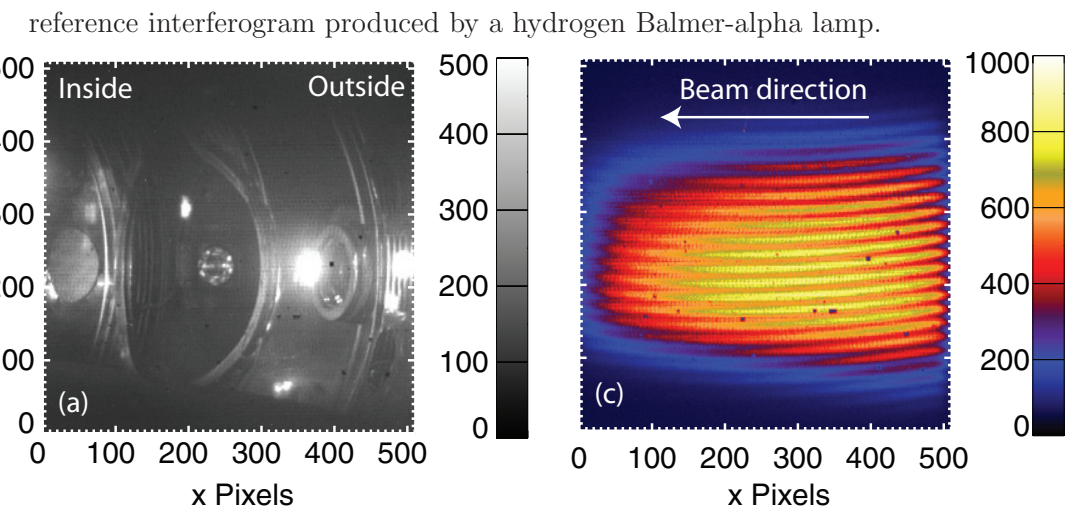

Figure 8. (a) Image of interior of TEXTOR vacuum vessel as recorded by the spectro-polarimeter. (b) Image of the neutral beam. Optical fibre cable imperfections are visible. Visible interference fringes indicate that the light is polarized. Successive images can be demodulated for the polarization orientation.

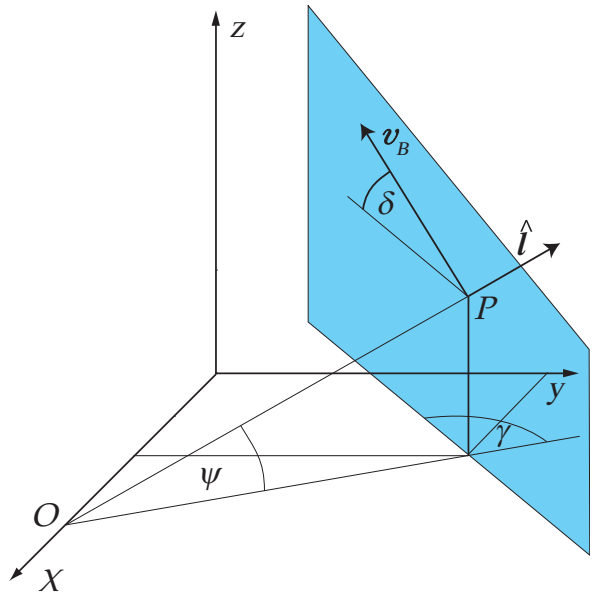

Figure 9. Diagram showing the relationship between the viewing line-of-sight $O P$ and beam velocity vector $\boldsymbol{v}_{B}$. The shaded section represents the top half of the neutral beam which is taken to be confined to a vertical plane.

$\boldsymbol{v}_{B}$ is the beam velocity vector and $v_{B}=\left|\boldsymbol{v}_{B}\right|$. A geometric analysis shows that the inner product of the unit vectors is given by

$$
\eta_{B}(\gamma, \psi) \equiv \hat{\boldsymbol{v}}_{B} \cdot \hat{\boldsymbol{l}}=\cos \gamma \cos \psi \cos \delta+\sin \delta \sin \psi
$$

where $\gamma$ and $\psi$ are the angles defining the intersection $O P$ of the view line-of-sight with the neutral beam sheet and $\delta$ is the vertical inclination angle of the neutral beam velocity at the intersection point $P$ (see figure 9).

Given the TEXTOR viewing geometry coordinates, it is possible to calculate the projection $\eta_{B}(\gamma, \psi)$ for different beam divergences. Figure 10(a) shows calculated contours of $\eta_{B}$ versus effective image angles $(\gamma, \psi)$ in the case of no beam divergence $(\delta=0)$. All images have been masked according to the intersection of the regions of non-negligible brightness in the observed beam emission and calibration images. Figure 10(b) shows the case for a simple (but unphysical) model in which $\delta$ increases linearly with an elevation angle $\psi$, spanning the angle range $\sim \pm 2.5^{\circ}$ vertically across the masked region. Finally, figure $10(\mathrm{c})$ shows contours of $\eta_{B}$ inferred from the measured Doppler interferometric phase shift image. The right edge of the experimental image exhibits some distortions due to demodulation artefacts generated at the periphery of the circular calibration image, while poorer fringe contrast degrades the phase-shift signal to noise ratio at the left region of the image. The experimental image clearly exhibits beam divergence and could, in principle, be unfolded for the beam velocity profile. In turn, this information can be used for the recovery of local magnetic field information from the image of the polarimetric tilt angle.
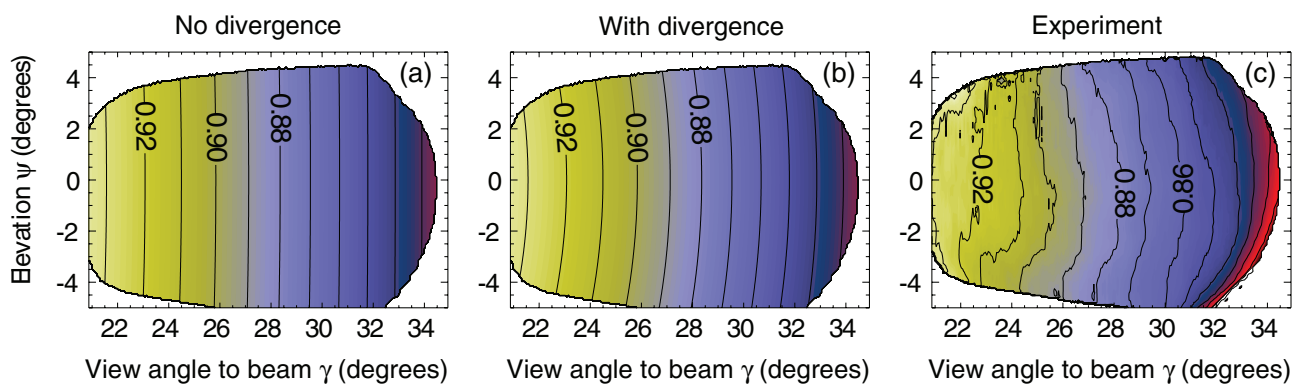

Figure 10. Calculated and measured contours of projected normalized velocity $\eta_{B}$ versus effective image angles $(\gamma$, $\psi)$. (a) No beam divergence (calculated), (b) beam angle $\delta$ increases linearly with $\psi$ spanning the range $\sim \pm 2.5^{\circ}$ and (c) measured contours of $\eta_{B}$. See text for discussion. 


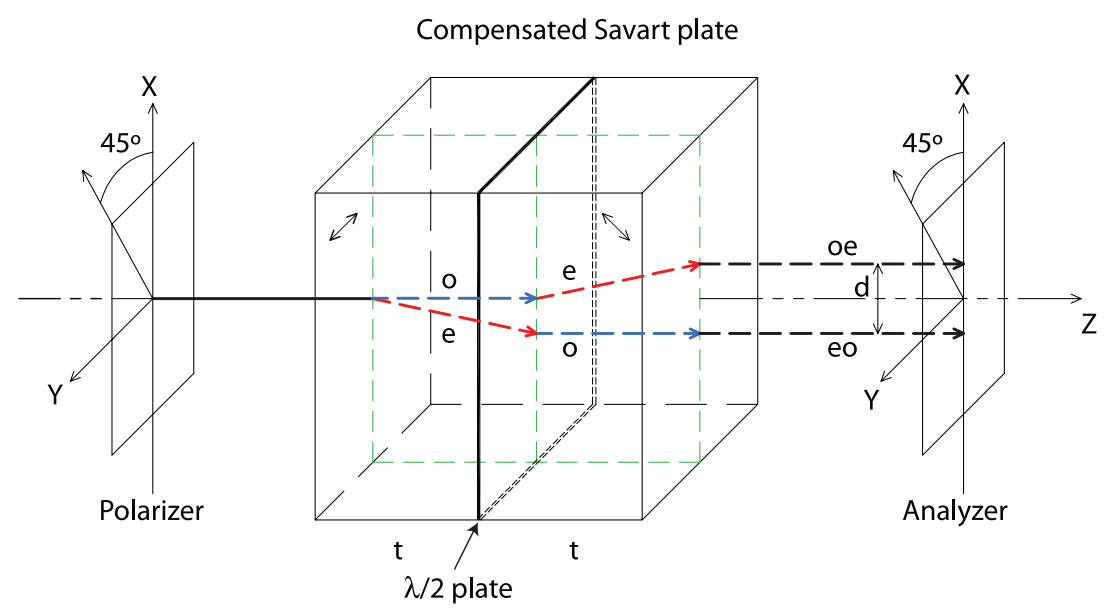

Figure A1. Showing the arrangement of birefringent plates and the associated ray paths for a compensated Savart plate prism.

\section{Acknowledgments}

This work is supported by International Science Linkages established under the Australian Government's innovation statement, 'Backing Australia's Ability'. I would like to thank colleagues M Creese, A Diallo for insightful discussions, B Blackwell for H-1 operational support and R Jaspers and $\mathrm{J}$ Chung for their assistance in performing the MSE measurements on TEXTOR.

\section{Appendix}

Here we review the construction and optical properties of various Savart prisms. A single birefringent plate (refractive indices $n_{e}$ and $n_{o}$ ) cut with its fast axis oriented at $45^{\circ}$ to the optical system $z$-axis in the $x-z$ principal plane gives rise to an $x$ displacement between the extraordinary and ordinary rays that exit the crystal. The optical path difference (OPD) between the separated, orthogonally polarized waves is given by [23]

$$
\Delta=t\left(A+\frac{a^{2}-b^{2}}{a^{2}+b^{2}} \cos \omega \sin \theta_{i}+\cdots\right)
$$

where $a=1 / n_{e}, b=1 / n_{o}, t$ is the plate thickness,

$$
A=\left(\frac{2}{a^{2}+b^{2}}\right)^{1 / 2}-\frac{1}{b},
$$

$\theta_{i}$ is the angle of incidence and $\omega$ is the angle between the plane of incidence and the principal section of the crystal plate. We see that the plate introduces a fixed phase delay and a phase shear proportional to the angle of incidence (for small angles). The lateral separation of the rays and its associated angledependent OPD gives rise to a pattern of interference fringes with phase fronts parallel to the $y$-direction when imaged by a lens through an appropriately oriented analyser.

A zero-nett-delay Savart shearing prism is formed by combining two such shear plates with their principal planes oriented orthogonally. The result of successive orthogonal displacements in the $x$ and $y$ directions produced by the double plate is a diagonal displacement. For a plate for total thickness $2 t$, the optical path difference in this case is [23]

$$
\Delta=t\left(\frac{a^{2}-b^{2}}{a^{2}+b^{2}}(\cos \omega+\sin \omega) \sin \theta_{i}+\cdots\right) .
$$

For wide field-of-view, the higher order angle-dependent terms indicated in equation (A.3) can result in interference fringes deviating from straight and parallel. This can be overcome using a field-widening compensation technique in which a half wave plate is interposed between the two Savart prisms, and oriented at $45^{\circ}$ to the direction of ray separation produced by the first prism [23]. This produces a $90^{\circ}$ polarization rotation such that the $o$ ray emerging from the first plate becomes the $e$ ray for the second and vice versa. The nett result is a vertical $(x)$ displacement of the rays as shown in figure A1 and an optical path difference between the $e o$ and $o e$ rays given approximately by

$$
\begin{aligned}
\Delta & =2 t \frac{a^{2}-b^{2}}{a^{2}+b^{2}} \cos \omega \sin \theta_{i} \\
& \approx \mathrm{d} \theta_{i}
\end{aligned}
$$

where $d$ is the nett spatial separation of the rays exiting the prism.

With the group delay for the Savart plate also given by equation (5), it is straightforward to show that the logarithmic derivative of the optical delay with respect to optical frequency is given by

$$
\left.\frac{\nu_{0}}{\tau_{0}} \frac{\partial \tau}{\partial v}\right|_{\nu_{0}}=\frac{4 a^{2} b^{2}(\beta-\alpha)}{a^{4}-b^{4}}
$$

where

$$
\begin{gathered}
\alpha=\left.\frac{\nu_{0}}{n_{\mathrm{o}}} \frac{\partial n_{\mathrm{o}}}{\partial v}\right|_{\nu_{0}} \\
\beta=\left.\frac{\nu_{0}}{n_{\mathrm{e}}} \frac{\partial n_{\mathrm{e}}}{\partial v}\right|_{\nu_{0}} .
\end{gathered}
$$




\section{References}

[1] Howard J 1999 Rev. Sci. Instrum. 70368

[2] Howard J 2002 Appl. Opt. 41197

[3] Howard J, Michael C, Glass F and Danielsson A 2003 Plasma Phys. Control Fusion 451143

[4] Howard J 2008 Plasma Phys. Control. Fusion 50125003

[5] Howard J, Michael C, Glass F and Cheetham A 2001 Rev. Sci. Instrum. 72888

[6] Chung J, Konig R, Klinger T and Howard J 2005 Plasma Phys. Control. Fusion 47919

[7] Howard J 2006 Rev. Sci. Instrum. 77 10F111

[8] Hamberger S, Blackwell B, Sharp L and Shenton D 1990 Fusion Technol. 17123

[9] Harris J et al 2004 Nucl. Fusion 44279

[10] Howard J, Diallo A, Jaspers R and Chung J 2010 J. Plasma Fusion Res. (at press)

[11] Born M and Wolf E 1980 Principles of Optics (Oxford: Pergamon)
[12] Goodman J 1985 Statistical Optics (New York: Wiley)

[13] Howard J, Glass F and Michael C 2007 J. Plasma Fusion Res. 2 S1014

[14] Howard J 1996 Plasma Phys. Control Fusion 38489

[15] Steel W 1967 Interferometry (Cambridge: Cambridge University Press)

[16] Michael C, Howard J and Blackwell B D 2001 Rev. Sci. Instrum. 721034

[17] Glass F, Howard J and Blackwell B 2005 IEEE Trans. Plasma Sci. $\mathbf{3 3} 472$

[18] Michael C, Howard J and Blackwell B 2004 Phys. Plasmas 114008

[19] Levinton F M et al 1989 Phys. Rev. Lett. 632060

[20] Wroblewski D et al 1990 Rev. Sci. Instrum. 613552

[21] Rice B W 1999 Fusion Eng. Design 34-35 135

[22] Creese M 2009 Honours Thesis Australian National University

[23] Francon M and Mallick S 1971 Polarization Interferometers (London: Wiley Interscience) 